\title{
How Much Contrast Media Can Be Used Safely in the Cardiac Catheterization Lab?
}

\author{
Richard Solomon \\ University of Vermont Medical Center, Burlington, VT, USA
}

\section{Keywords \\ Acute kidney injury · Contrast media · Percutaneous coronary intervention}

In this issue of the journal, a group from Boston reassesses the maximum amount of contrast dose (MACD) at which the risk of acute kidney injury (AKI) increases in patients undergoing coronary interventions [1]. Acute renal injury following coronary interventions remains a serious adverse event that is associated with long-term worsening of kidney function and cardiovascular morbidity and mortality. As there is no effective therapeutic to reverse the injury once it occurs, prevention is the cornerstone of managing patients. Current guidelines from the primary cardiology societies (AHA, ACC, and SCAI) recommend a 3-pronged approach to the prevention of AKI [2]. First is to assess individual patient risk of developing AKI. There are a number of risk assessment tools, but all rely on knowledge of baseline kidney function and most include the amount of contrast media administered. A second preventative approach is to ensure that patients get "adequate hydration." Although this is not defined further, most cardiologists and nephrologists take this to mean correction of any preexisting volume depletion by administering isotonic saline intravenously before, dur- ing and after the procedure and promoting a vigorous urine output to dilute out the contrast in the urinary space. The third arm of the prevention strategy is to limit the amount of contrast given for the procedure. Sampling of cardiologist's practices reveals a very large spectrum of contrast use for PCI reflecting differing patterns of adherence to this third guideline [3].

A number of techniques and devices have been developed to limit the exposure of the kidney to contrast, including avoidance of unnecessary LV grams, use of intravascular ultrasound, automated contrast injectors, pressure sensitive diverters, coronary sinus aspiration, use of the radial artery approach with small-bore catheters, and staging complex procedures [4]. For the most part, these approaches can be established in advance of the procedure and protocolized. However, during the procedure, it is also necessary to track how much contrast has been administered and have a MACD that represents the dose at which the risk of AKI increases significantly in that individual.

It is this latter determination of AKI risk that has been the subject of multiple studies. To make the calculation of a maximum dose relevant to the individual patient, the majority of the proposed equations use baseline kidney function as a key factor. This is consistent with the guide- 
line recommendations to stratify risk based on baseline kidney function. It also makes pharmacokinetic sense. Contrast is freely filtered and its half-life increases as GFR decreases. Thus, a lower GFR means contrast remains for a longer period of time in the circulation. With a decrease in GFR, there is also a parallel decrease in the number of nephrons. As a result, each nephron in a patient with CKD has a larger load of contrast to filter and excrete. Thus, the exposure burden (time and amount filtered) is greater per nephron. Some calculations also use body weight, a surrogate for volume of distribution $\left(V_{\mathrm{d}}\right)$ of the contrast and the concentration of contrast in the blood, as an additional component. By keeping the total amount of contrast administered below this MACD, the risk of $\mathrm{AKI}$ is mitigated. In the original description of the MACD, the authors suggested that contrast dose should be limited to $5 \times$ body weight $(\mathrm{kg})$ divided by the serum $\mathrm{Cr}(\mathrm{mg} /$ dL) [5].

Hatter and colleagues [1] collected data from a single center in Boston over 10 years involving 3,605 patients who underwent percutaneous coronary interventions (PCI). The investigators sought to determine the MACD using baseline eGFR and body weight.

The original MACD equation was developed in patients with CKD $(\mathrm{Cr} \geq 1.8 \mathrm{mg} / \mathrm{dL})$ undergoing PCI with high osmolar contrast and in whom the definition of AKI was a $\mathrm{Cr}$ rise of $\geq 1.0 \mathrm{mg} / \mathrm{dL}$ [5]. It was chosen arbitrarily and not determined by modern statistical approaches using ROC curves and deriving the point of best sensitivity and specificity. Only 115 patients were studied of whom 8 developed AKI. Cr clearance was estimated from the Cockcroft-Gault equation rather than eGFR as this was pre-MDRD and CKD-EPI equations. The approach described in the current report is more generalizable as it includes all patients undergoing PCI. As these patients were exposed to iso-osmolar contrast media and given protocolized intravenous fluid before and after contrast exposure, it is more consistent with modern practice.

One might expect that the risk of AKI would be lessened by our modern contrast agents and prophylactic strategies. Indeed, the incidence of moderate to severe AKI in the current study was 1.9 versus $7.0 \%$ in the original data. The authors found that the MACD was much lower than that reported in the original description by about half ( 2.5 vs. 5.0$)$. The difference in the constant is likely explained by differences in the definition of AKI and the more rigorous statistical approach.

There are a number of concerns around setting a precise MACD. First, is the assumption that all AKI following a coronary intervention is the result of the contrast.
All of the literature on MACD uses retrospectively collected data. There is no adjudication regarding other potential causes of AKI such as atheromatous emboli, and hemodynamic insults. The number of AKI events is thus an overestimation of the true rate of contrast associated AKI. When the total number of AKI events is small, even a few cases of AKI not related to contrast could bias the statistics. Second, the volume of contrast may itself be a surrogate for some of these other causes of AKI. For example, more contrast will be given when the number of stents placed increases and/or the deployment of the stents is more challenging. Either situation might require more manipulation of catheters increasing the risk of atheromatous dislodgement. Third, it is unlikely that "one size fits all." Indeed, as the authors of the current report show, the coefficient $(K)$ is different in different subgroups of patients. While most of these differences are not statistically significant, it is interesting that $K$ is highest (i.e., you can give more contrast for a given GFR) in those with reduced GFRs at baseline. This is counterintuitive based upon the pharmacokinetics discussed earlier. It may be that risk of AKI is so high in CKD to begin with, which you have to give a huge dose to make the risk worse.

Where does this leave us? Minimizing the dose of contrast is clearly important but choosing any particular MACD should be approached with some skepticism. A survey of 506 cardiology practices found that only $62 \%$ of practices measured the amount of contrast administered [6]. Indeed, it may be more important to have any MACD as part of a protocol than to have none at all [7]. The current article is important because it gives us a target dose that can be monitored during the procedure.

\section{Conflict of Interest Statement}

The authors have no conflicts of interest to disclose.

\section{Funding Sources}

The author did not receive any funding.

\section{Author Contributions}

The author has written the commentary. 


\section{References}

1 Hattar L, Assaker JP, Aoun J, Price LL, Carrozza J, Jaber BL. Revising the maximal contrast dose for predicting acute kidney injury following coronary intervention. Am J Nephrol. 2021.

2 Wright RS, Anderson JL, Adams CD, Bridges CR, Casey DE Jr, Ettinger SM, et al. 2011 ACCF/AHA focused update of the guidelines for the management of patients with unstable angina/non-ST-elevation myocardial infarction (updating the 2007 guideline): a report of the American College of Cardiology Foundation/American Heart Association task force on practice guidelines developed in collaboration with the American College of emergency physicians, society for cardiovascular angiog- raphy and interventions, and society of thoracic surgeons. J Am Coll Cardiol. 2011; 57(19):1920-59.

3 Amin AP, Bach RG, Caruso ML, Kennedy KF, Spertus JA. Association of variation in contrast volume with acute kidney injury in patients undergoing percutaneous coronary intervention. JAMA Cardiol. 2017;2(9):1007-12.

4 Almendarez M, Gurm HS, Mariani J Jr, Montorfano M, Brilakis ES, Mehran R, et al. Procedural strategies to reduce the incidence of contrast-induced acute kidney injury during percutaneous coronary intervention. JACC Cardiovasc Interv. 2019;12(19):1877-88.

5 Cigarroa RG, Lange RA, Williams RH, Hillis LD. Dosing of contrast material to prevent contrast nephropathy in patients with renal disease. Am J Med. 1989;86(6 Pt 1):649-52.

6 Prasad A, Sohn A, Morales H, Williams K, Bailey SR, Levin D, et al. Contemporary practice patterns related to the risk of acute kidney injury in the catheterization laboratory: results from a survey of Society of cardiovascular angiography and intervention (SCAI) cardiologists. Catheter Cardiovasc Interv. 2017; 89:383-92.

7 Brown JR, Solomon RJ, Sarnak MJ, McCullough PA, Splaine ME, Davies L, et al. Reducing contrast-induced acute kidney injury using a regional multicenter quality improvement intervention. Circ Cardiovasc Qual Outcomes. 2014;7(5):693. 